\title{
THE EFFECTS OF MODEL ASSUMPTIONS ON THE DYNAMIC IMPEDANCE FUNCTIONS OF A SHALLOW FOUNDATION
}

\section{Johan Lind Östlund $^{1,2}$, Andreas Andersson ${ }^{1,3}$, Mahir Ülker-Kaustell ${ }^{1,2}$, and Jean-Marc Battini $^{1}$} \author{
Stockholm, Sweden \\ ${ }^{2}$ Tyréns AB \\ Stockholm, Sweden \\ ${ }^{3}$ Swedish Transport Administration \\ Solna, Sweden
}

${ }^{1}$ Division of Structural Engineering and Bridges, KTH Royal Institute of Technology

e-mail: joost@kth.se, \{andreas.andersson, mahir.ulker, jean-marc.battini\}@byv.kth.se

Keywords: Dynamic impedance functions, Permanent load, Dynamic soil-structure interaction, Soil dynamics.

\begin{abstract}
The effects of model assumptions on the dynamic impedance functions of shallow foundations are investigated using finite elements. The shallow foundations are considered massless and rigid and are positioned in strata of frictional soil on top of bedrock. Two studies are established. The first investigates the combined effects of model assumptions including the variation of modulus with depth, the embedment of the foundation, and permanent load acting on the foundation. The second study is a parametric analysis investigating the effects of permanent load considering strata with varying soil modulus coefficients and with varying soil depths. Functions describing the small-strain modulus and the modulus reduction of frictional soil are used in an iterative process to update the spatial modulus distribution of the soil due to the permanent load. The results show that model assumptions can have a large effect on the impedance functions. The static stiffness coefficients vary substantially, in some instances by more than $100 \%$. The impedance functions, normalized with the static stiffness coefficients, match each other well in the frequency range below the fundamental frequency. However, in the frequency range above the fundamental frequency, the normalized impedance functions differ substantially from each other. Further, the results show that the effect of the permanent load is largest in the case of shallow and stiff soil strata, both regarding normalized impedance functions as well as the static stiffness coefficient, which was increased up to $67 \%$. The variation in fundamental frequency is however small.
\end{abstract}




\section{INTRODUCTION}

In the design of high-speed railway bridges, a dynamic assessment is required. The boundary conditions of these bridges are in many design situations modeled with fixed supports. However, the flexibility of the supports, due to the dynamic soil-structure interaction (SSI), may significantly change the dynamic properties of the bridges. One method of considering the SSI is by decoupling the models and calculating impedance functions. Instead of computing the complete soil-foundation-structure system, the model is decoupled and impedance functions are calculated from the soil-foundation subpart before being applied to the structure. This sub-structuring of the system enables faster calculations, especially at train loading analysis, as compared to the calculation of the complete system.

The impedance function is commonly expressed in complex numbers:

$$
Z(\omega)=k_{d}(\omega)+i \omega c_{d}(\omega)
$$

where $k_{d}$ is the dynamic stiffness coefficient, $c_{d}$ is the dashpot coefficient, $\omega$ is the cyclic frequency of excitation, and $i=\sqrt{-1}$.

Impedance functions have been published in many journal papers and are compiled in handbooks to give structural engineers simple tools to take SSI into account in design (see e.g. [1] or [2]). Although the studies given in the literature fully explore the effects of one or two of the specific features in each paper, when it comes to combinations of features that are not given in the literature, it may be difficult for the engineer to find the relevant model assumptions to use in a specific project and what consequences to expect. To fill that gap, this paper investigates the effects of the embedment of the foundation, the variation of the modulus with the depth, and the depth of the stratum on bedrock in different combinations. Further, the effects of applying a permanent load to the soil-foundation system and updating the spatial distribution of the modulus before performing linear dynamic analyzes are introduced.

The purpose of this paper is to show the effects of model assumptions on the dynamic impedance functions of shallow foundations. The article aims at filling the gap in the literature by investigating the combined effects of model assumptions from a practical point of view. Two main objectives of the study are defined. The first was to study the implications of model assumptions, including the embedment of the foundation, the variation of the modulus with the depth, and the permanent load. Impedance functions calculated from the models with varying levels of idealization are compared in the paper. The second objective was to quantify the influence of permanent load in relation to the soil modulus coefficient and stratum depth. A parametric study was performed for this purpose. To fulfill the objectives, numerical models using finite elements (FE) were created. The shallow foundation was assumed to be rigid and massless and was given a fixed geometry. The soil strata consisted of frictional soil supported by bedrock. Whereas the permanent load of the structure was included in the soil-foundation FE model in order to consider its effect on soil properties, the mass of the structure was not. This mass will be taken into account when the impedance functions are attached to the structure. The spatial variation of the modulus of elasticity caused by the permanent load was updated for each element, considering functions for the low-strain modulus and modulus reduction. Linear dynamic analyses were then performed on the updated models, and the impedance functions were calculated. The dynamic loading was assumed to affect the soil within the linear elastic range, and the impedance functions are valid in the serviceability limit state.

The soil and foundation parameters were chosen in the context of high-speed railway (HSR) bridges and Swedish soil conditions. Swedish geology is dominated by glacial soil masses 
on top of high-quality bedrock [3]. At many locations, lodgment moraine overlays bedrock, possibly covered by clay or silt layers. For shallow strata consisting of frictional soil, shallow foundations may be suitable for HSR bridges. Such soil deposits are limited in depth, and the maximum depth considered in this study was assumed to be $8 \mathrm{~m}$. The embedment depth of a foundation is typically determined by the frost-free depth, which in Sweden is considered at 1.1-2.5 m.

\section{SOIL PROPERTIES}

\subsection{Small-strain shear modulus}

The small-strain shear modulus of soil has been studied empirically by several authors for soil types ranging from cohesive to frictional, and many suggestions for formulas have been proposed [4-6]. Seed and Idriss [7] suggested a simplified formula for the small-strain shear modulus of frictional soil:

$$
G_{0}=K_{0}\left(\sigma^{\prime}\right)^{d}
$$

where $K_{0}$ is the soil modulus coefficient, $d$ is the stress exponent, and $\sigma^{\prime}$ is the mean effective stress. $K_{0}$ and $d$ are soil type-specific constants. The Swedish design recommendations [8] have adopted this formula and adjusted it for Swedish conditions. In the recommendations, $K_{0}$ ranges from 15,000 to 30,000 where the first value corresponds to sand and the latter to crushed fill material. Furthermore, $d=1 / 2$, and the stress is inserted in $\mathrm{kPa}$.

The mean effective stress $\sigma^{\prime}$ can be subdivided into two additive parts. For frictional soil, the stress in the undisturbed in situ soil is commonly estimated by the confined effective stress based on the coefficient of lateral earth pressure [9]:

$$
\sigma_{0}^{\prime}=\sigma_{v}^{\prime} \frac{1+2 k}{3}
$$

where this approximate relationship can be used:

$$
k=1-\sin \left(\phi^{\prime}\right)
$$

Here, $\sigma_{v}^{\prime}$ is the effective vertical stress, $k$ is the coefficient of lateral earth pressure, and $\phi^{\prime}$ is the drained friction angle. Assuming no groundwater, the effective vertical stress $\sigma_{v}^{\prime}$ can be calculated with $\rho g z$ where $\rho$ is the density, $g$ is the gravity constant, and $z$ is the depth from the soil surface. External load adds to $\sigma^{\prime}$ by the mean effective stress

$$
\sigma_{\mathrm{m}}^{\prime}=\frac{\sigma_{x}^{\prime}+\sigma_{y}^{\prime}+\sigma_{z}^{\prime}}{3}
$$

where $\sigma_{x}^{\prime}, \sigma_{y}^{\prime}$, and $\sigma_{z}^{\prime}$ are the effective normal stresses. Finally, the resulting stress becomes

$$
\sigma^{\prime}=\sigma_{0}^{\prime}+\sigma_{\mathrm{m}}^{\prime}
$$

\subsection{Normalized modulus reduction}

The reduction of modulus and increase of material damping ratio due to loading have been evaluated empirically in numerous studies for soil gradations ranging from cohesive to frictional soils [10-12]. In recent decades, researchers have aimed at collecting data from empirical studies and describing nonlinear relationships using statistical tools. In [13], a statistical analysis of empirical data from resonant column tests was conducted. This produced a set of 18 dimensionless parameters describing the normalized modulus reduction curves and material damping ratio 
curves. The 18 parameters can be chosen to get either mean values or variances of the curves, and their values are dependent on the soil type. The normalized modulus reduction curve is calculated with

$$
\frac{G}{G_{0}}=\frac{1}{1+\left(\frac{\gamma}{\gamma_{\mathrm{r}}}\right)^{a}}
$$

where $\gamma$ is the current shear strain, $\gamma_{\mathrm{r}}$ is the reference shear strain, and $a$ is the curvature coefficient. The formulas for $\gamma_{\mathrm{r}}$ and $a$ are found in [13] and require, in addition to the 18 dimensionless parameters, the plasticity index, over-consolidation ratio, and stress in the soil. Only soils with gradations ranging from clay to sand were included. However, the difference in normalized modulus reduction curves between different gradations of frictional soils is relatively small $[12,14]$. A comparison between the mean normalized modulus reduction curve of gravelly soils by Rollins et al. [12] and the corresponding curve for sand in [13] shows good agreement.

The shear strain can be calculated from the effective octahedral shear strain [15],

$$
\gamma=\frac{\alpha}{3} \sqrt{\left(\varepsilon_{x}-\varepsilon_{y}\right)^{2}+\left(\varepsilon_{x}-\varepsilon_{z}\right)^{2}+\left(\varepsilon_{y}-\varepsilon_{z}\right)^{2}+6\left(\gamma_{x y}^{2}+\gamma_{x z}^{2}+\gamma_{y z}^{2}\right)}
$$

where $\varepsilon_{i}$ and $\gamma_{i j}$ are components of the strain vector and $\alpha=0.65$. This shear strain measure has been used in previous studies $[16,17]$.

\section{NUMERICAL STUDIES}

A fixed geometry of the foundation was used in all numerical simulations, and an overview of the FE model including the dimensions of the foundation is presented in Figure 1. The foundation was regarded as massless and rigid. The dimensions of the foundation are as follows: $L=9.4 \mathrm{~m}, B=3.3 \mathrm{~m}$, the thickness of the slab is $1.0 \mathrm{~m}$, the length of the support wall is 7.0 $\mathrm{m}$, and the thickness of the support wall is $0.9 \mathrm{~m}$. $H_{1}$ is the soil depth underneath the bottom of the foundation, $\mathrm{H}_{2}$ is the embedment depth and $R$ is the radius of the FE model. The FE model is further described in section 4.1.

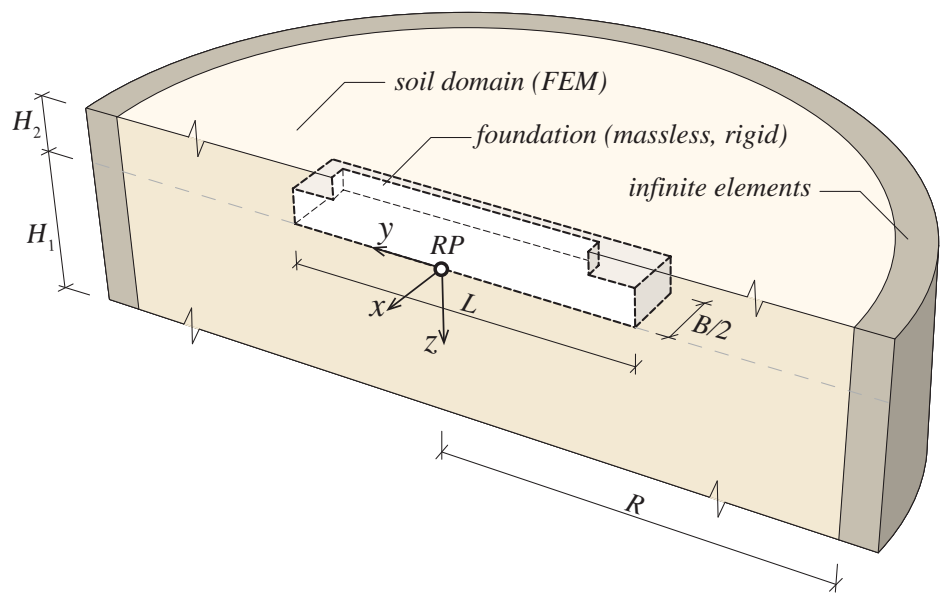

Figure 1: A cross-section of the finite element model.

The material properties of the soil were chosen from typical values of frictional lodgment moraine [18] and were assumed constant in all calculations. The following material properties were chosen for all cases: material damping ratio $\xi=2.0 \%$; density $\rho=2000 \mathrm{~kg} / \mathrm{m}^{3}$; Poisson's 
ratio $\nu=0.25$; friction angle $\phi^{\prime}=45 \mathrm{deg}$; stress exponent $d=1 / 2$, and gravity constant $g=$ $9.81 \mathrm{~m} / \mathrm{s}^{2}$.

The permanent load was applied as a force $P$. In order to relate the load to the size of the foundation, the load is presented as a pressure $q$ obtained from dividing the force $P$ with the foundation's bottom surface area. The following values were taken: $q=(0,220,500)$ $\mathrm{kPa}$. According to the Swedish design requirements, the upper limit on the pressure of shallow foundations on top of lodgment moraine on bedrock is $600 \mathrm{kPa}$ [19].

The modulus at the surface of the stratum goes toward zero due to Eq. 2. This can cause poor conditioning of the elements because elements with high modulus are next to elements of low modulus, which can be particularly sensitive in the calculation of impedance functions from surface foundations. To avoid this, a lower limit for the modulus corresponding to the value at a depth of $0.2 \mathrm{~m}$ was chosen.

\subsection{Model assumptions study}

The effects of the model assumptions on the impedance functions were studied by investigating four cases as shown in Figure 2. It was decided that the fundamental frequency of all cases should be the same. This choice was made based on the fact that the behavior of the soil-foundation system is essentially different at frequencies below compared to above the fundamental frequency of the soil stratum, and fixing it was considered to be the best way to compare the models. In order to get the fundamental frequency of the homogeneous stratum to match the fundamental frequency of the stratum with a modulus varying with depth, the modulus value of the homogeneous soil stratum can be obtained by calculating an equivalent depth $z_{\text {eq }}[20,21]$. $z_{\text {eq }}$ determines the depth at which the equivalent modulus value $G_{\text {eq }}$ of the modulus varying with the depth $G\left(K_{0}, \sigma_{0}^{\prime}\right)$ is taken from. $G_{\text {eq }}$ is then applied to the homogeneous stratum and the equivalent wave speed $v_{\text {eq }}$ gives the fundamental period $T=4 H / v_{\text {eq }}$, where $H$ is the total stratum depth. In this work, since $d=1 / 2$ in Eq. 2, the equivalent depth was calculated to $z_{\text {eq }}=0.64 H$.

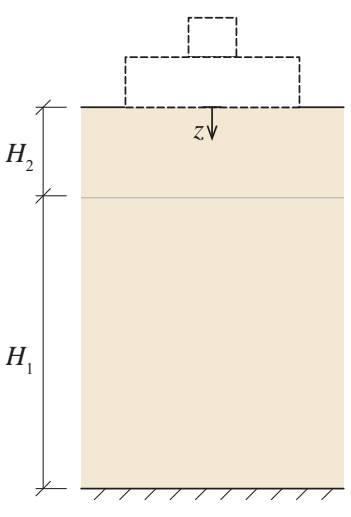

a)

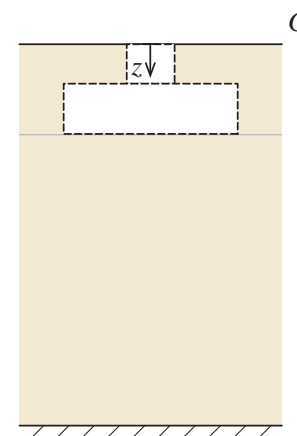

b)

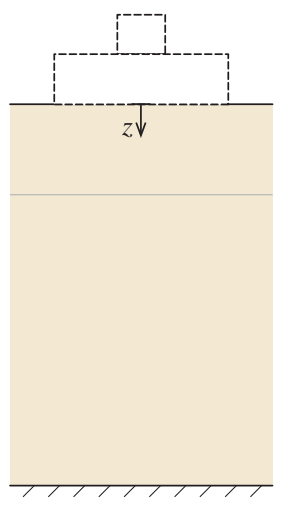

c)

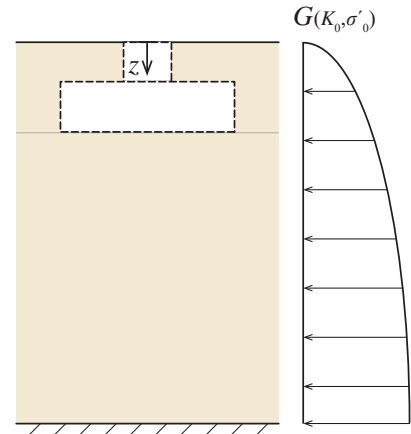

d)

Figure 2: Cases considered in the model assumptions study.

The model assumption cases had the following properties:

- Case (a): A surface foundation on a stratum of homogeneous soil. No permanent load.

- Case (b): An embedded foundation in a stratum of homogeneous soil. No permanent load. 
- Case (c): A surface foundation on a stratum with modulus varying with the depth. Includes permanent load $P$.

- Case (d): An embedded foundation in a stratum with modulus varying with the depth. Includes permanent load $P$.

The following parameters were chosen for all the models: $H_{1}=8 \mathrm{~m}$ and $K_{0}=30,000$, corresponding to a deep soil stratum consisting of stiff lodgment moraine. The embedment depth was fixed at $H_{2}=1.6 \mathrm{~m}$. The modulus of Case (a) and Case (b) was calculated to $G_{\text {eq }}=240$ $\mathrm{MPa}$.

\subsection{Parametric study}

The effect of the permanent load was studied for the strata with varying depth $H_{1}$ and soil modulus coefficient $K_{0}$ in the parametric study. All the models had the configurations of Case (d) in Figure 2. Eighteen combinations were analyzed based on the following parameters: $q=$ $(0,220,500) \mathrm{kPa}, H_{1}=(2,4,8) \mathrm{m}$, and $K_{0}=(15,000,30,000)$. The embedment depth was fixed at $H_{2}=1.6 \mathrm{~m}$.

\section{NUMERICAL MODEL}

\subsection{FE model}

The numerical model of the soil-foundation system was created in the commercial FE software Abaqus [22], and an overview of the FE model is presented in Figure 1. The soil domain was created as a disk with a thickness equal to the stratum depth, and the bedrock was made infinitely stiff by constraining the bottom surface of the disk against translations. A void within the soil domain was created with the geometry of the embedded foundation. Then, rigid connections were applied to the surfaces of the soil facing the void linking them to a reference point (RP) positioned at the midpoint on the bottom surface. In the case of a surface foundation, no void was created and the single surface at the interface between soil and foundation was rigidly connected to the RP. Loading was applied and output was taken at the RP. The damping of the soil was applied as rate-independent structural damping which is incorporated in the complex modulus as $\boldsymbol{K}^{*}=\boldsymbol{K}(1+i 2 \xi)$, where $\boldsymbol{K}$ is the stiffness matrix.

Quadratic tetrahedral solid elements were used. The mesh size at the center of the soil domain at the foundation was $0.25 \mathrm{~m}$. From the center, the mesh size was gradually enlarged along the radius toward the boundary of the model. The radius of the model was $R=200 \mathrm{~m}$. At the boundary, the mesh size was $20 \mathrm{~m}$. Due to the gradual enlargement of the elements, the computational time was reduced without causing spurious waves that can occur due to large size differences between elements. Infinite elements [23] were attached to the outer surface boundary of the model domain.

\subsection{Calculation procedure}

The updating of the G-modulus in each of the elements due to permanent load and the calculation of impedance functions were performed as follows:

1. Initially, the unloaded modulus was assigned: Calculate $\sigma_{0}^{\prime}$ (Eq. 3) and $G_{0}$ (Eq. 2). In this state, $G / G_{0}=1$ (Eq. 6), and the shear modulus is $G=G_{0}$.

2. Apply permanent load $P$ to the RP in Figure 1 and run a static analysis. 


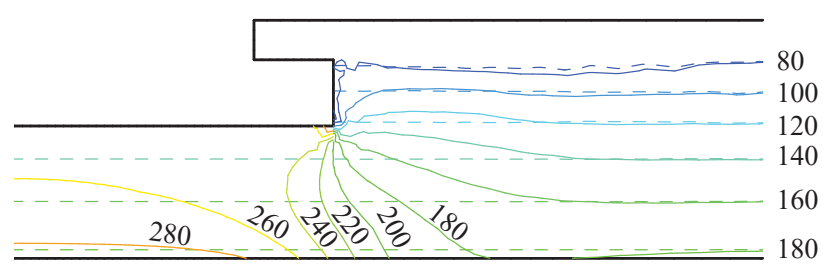

a)

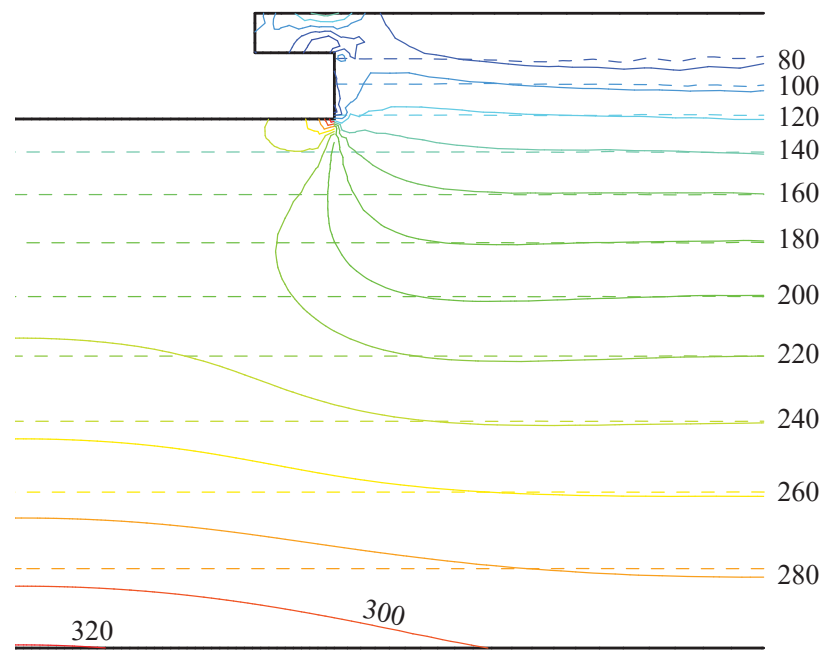

b)

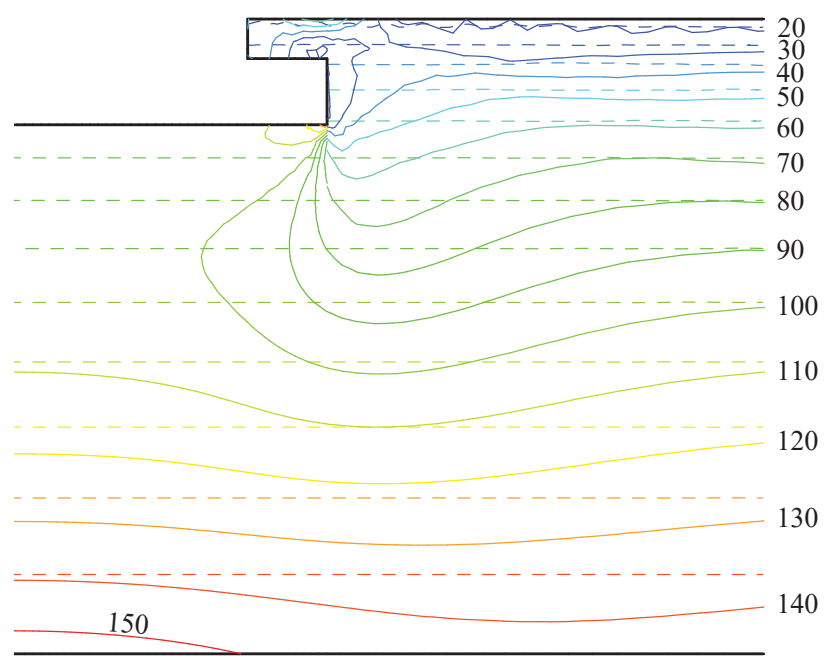

c)

Figure 3: Updating of the shear modulus distribution due to permanent load. Values given in MPa. a) $H_{1}=2 \mathrm{~m}, K_{0}=30,000$, b) $H_{1}=8 \mathrm{~m}, K_{0}=30,000$ and c) $H_{1}=8 \mathrm{~m}, K_{0}=15,000$. Dashed lines: Unloaded soil. Solid lines: Updated soil.

3. Successively calculate: $\sigma_{0}^{\prime}$ (Eq. 3), $\sigma_{m}^{\prime}$ (Eq. 4), $\sigma^{\prime}$ (Eq. 5), $G_{0}$ (Eq. 2), $\gamma$ (Eq. 7), and $G$ (Eq. 6).

4. Repeat steps 2 and 3 until convergence of the distribution of shear modulus $G$ is reached.

5. Calculate impedance functions.

Direct steady-state calculations were performed in order to obtain the impedance functions. 
Harmonic unit loads were individually applied at the RP displayed in Figure 1 to each of the six degrees of freedom in a given frequency range. The compliance functions $U$ were obtained from the model. The impedance functions were then calculated by the inverse of the compliance functions $Z=U^{-1}$. Only the values of the diagonal of the impedance function matrix were analyzed.

The converged distributions of the shear modulus for three of the models are shown in Figure 3 , and the cuts are taken along the $y-z$ plane. The models were configured as in Section 3.2 with $q=220 \mathrm{kPa}$. An increase of the permanent load results in two opposite effects on the modulus: an increase of $G_{0}$ due to the increase of $\sigma^{\prime}$ and a reduction by $G / G_{0}$ due to the increase of $\gamma$. In the volume between the foundation and the bedrock, the permanent load caused an increase in the modulus. The influence is large for shallow stratum depths. Just outside of this volume, the modulus can be either reduced or increased from permanent load depending on the stress-shear strain interaction.

\section{RESULTS}

The real part of the impedance function is denoted with $\operatorname{Re}\left(Z_{j}\right)$, whereas the imaginary part is denoted with $\operatorname{Im}\left(Z_{j}\right)$. The degrees of freedom are indicated by the subscript $j$ and the coordinate axes are defined in Figure 1. The compliance functions, $\left|U_{j}\right|$, are denoted in the same way as the impedance functions and are given as absolute values. All functions are presented with the dimensionless frequency $a_{0}=\omega w / v_{s, \text { eq }}$ where $w=B / 2$ and $v_{s, \text { eq }}=\sqrt{G_{\text {eq }} / \rho}$ is the equivalent shear wave velocity of the strata (without the influence of the permanent load). Impedance functions are normalized with the static stiffness coefficients $k_{j, \text { stat }}$, and the compliance functions are normalized by the static displacements $u_{j \text {,stat }}$.

\subsection{Model assumptions study}

The normalized impedance functions from the model assumptions study are presented in Figure 4 including the $x$ and $z$ degrees of freedom. The corresponding static stiffness coefficients are given in Figure 5. The following observations are made:

- The static stiffness coefficients range from 3 to $9 \mathrm{GN} / \mathrm{m}$ depending on the model case. The embedment of the foundation increases the static stiffness coefficient as compared to the static stiffness coefficient of the corresponding surface foundation, as shown by comparing Case (a) to Case (b), and Case (c) to Case (d). This is explained by the reduced distance to the bedrock. Strata with a modulus varying with the depth have lower static stiffness coefficients than strata with homogeneous modulus. This is evident when comparing Case (a) to Case (c) and Case (b) to Case (d). With increasing permanent load, the values of the static stiffness coefficients in Cases (c) and (d) are increased but they do not exceed the static stiffness coefficients of the homogeneous cases of (a) and (b).

- In the pre-resonance state, i.e. before wave propagations are initiated, the impedance functions are proportional to the static properties of the soil-foundation system. The normalized impedance functions can be described by a single degree of freedom system. The real part of this system is $\left(k_{j \text {,stat }}-\omega^{2} m_{j \text {,stat }}\right) / k_{z \text {,stat }}$, where $m_{j \text {,stat }}$ is the mass of the system, and the imaginary part is constant and is equal to $i 2 \xi$. The normalized impedance functions are thus very similar in the pre-resonance frequency range, however, they differ substantially at higher frequencies. In the post-resonant frequency range, the impedance is highly influenced by wave propagations and the single degree of freedom system can 

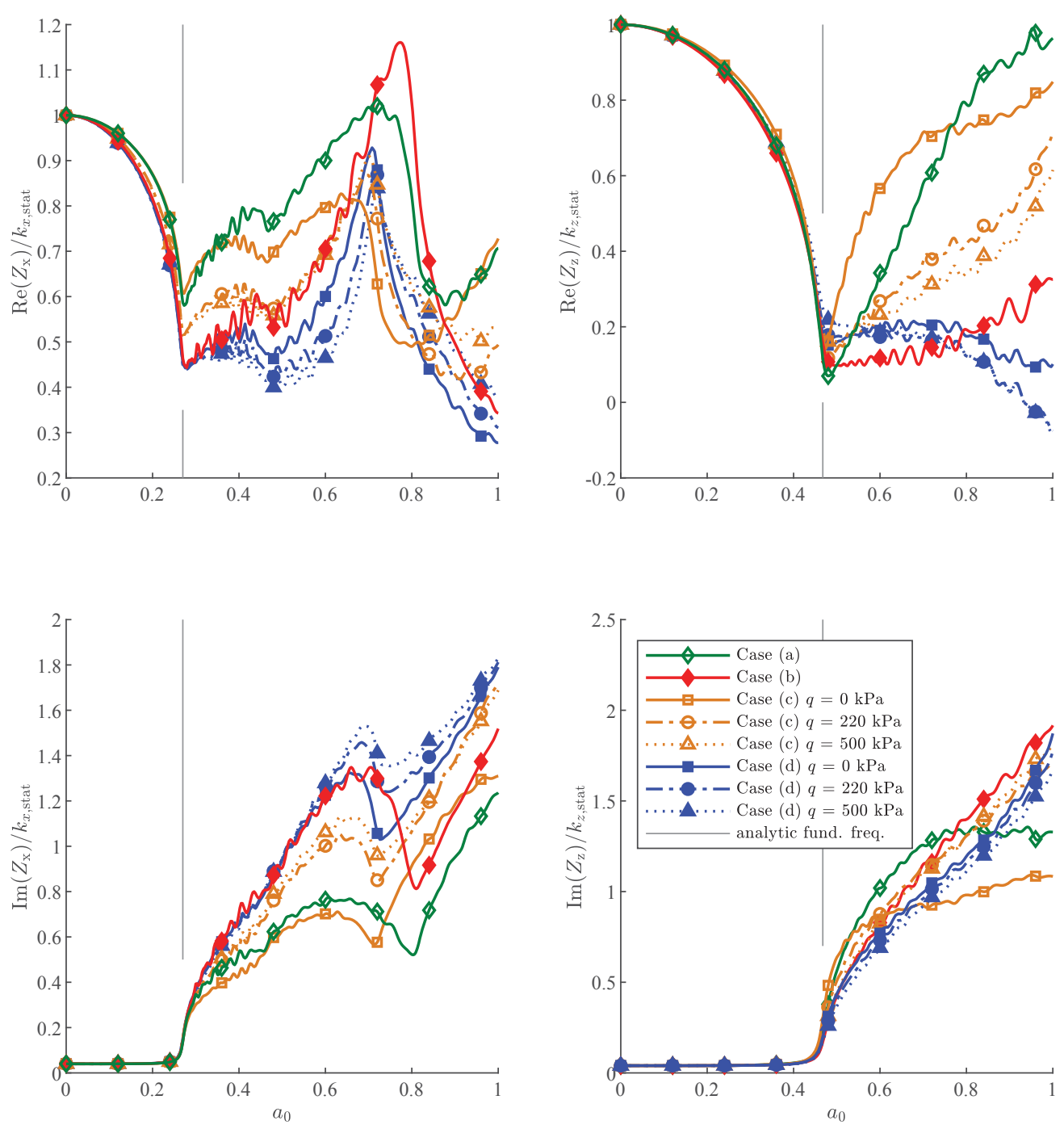

Figure 4: Normalized impedance functions from the model assumptions study.

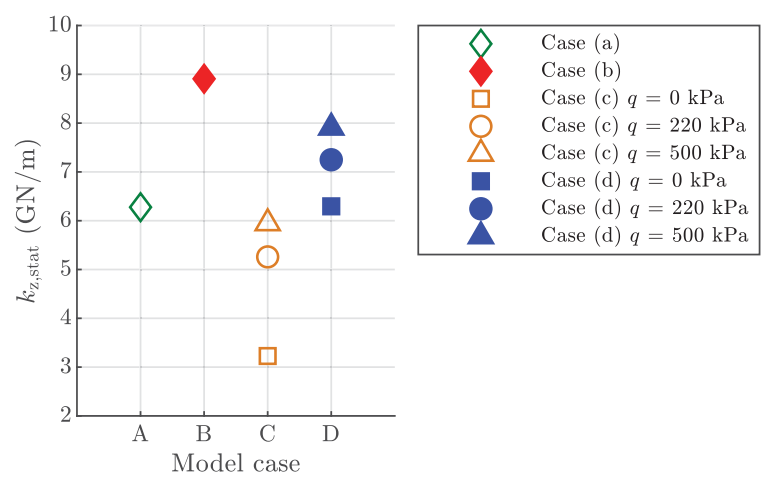

Figure 5: Static stiffness coefficients of the four model cases. 
no longer describe the impedance functions. The imaginary part is increased significantly due to the radiation damping, and the differences in impedance between the cases are large.

- All cases had the same fundamental frequency which was also the same as the analytical fundamental frequency of the soil strata [20]. The fluctuations in impedance that can be observed for all cases at $a_{0} \approx 0.7$ in the $x$-direction are due to the second mode of vibration of the soil, which for a homogeneous stratum has the natural frequency of three times the fundamental frequency according to $T_{n}=(4 H / v) /(2 n-1)$ were $v$ is the wave speed and $n$ is the mode number. The natural frequencies of the second mode for the strata with modulus varying with the depth are somewhat lower than the natural frequencies of the strata with homogeneous soil. The fundamental frequencies of the strata are not changed by the local influence that the permanent loads have on the soil moduli.

- For surface foundations on top of strata with modulus varying with the depth, as in Case (c), very low static stiffness coefficients are obtained with $q=0$. This shows the consequences of omitting permanent load in combination with a very low modulus at the surface of the model. Adding the permanent load to Case (c) increases the static stiffness coefficient considerably. This effect is also shown for the normalized impedance functions, where an increase of the permanent load leads to a decrease of the real part of the impedance and an increase of the imaginary part.

\subsection{Parametric study}

Normalized impedance functions in the $z$-direction are presented in Figure 6. The static stiffness coefficients of each case, normalized with the static stiffness coefficients with $q=0$ $\mathrm{kPa}$, are given in Figure 7. The static stiffness coefficients with $q=0 \mathrm{kPa}$ are given in Table 1 as a reference. Normalized compliance functions are presented in Figure 8. The following observations are made:

- For most cases, the static stiffness coefficient increases with the permanent load, in particular for shallow strata with a high soil modulus coefficient, see Figure 7. This can be explained by the high pressure and the small shear strain that mainly increase the modulus, see Figure 3 (a)-(b). The maximum increase of the static stiffness coefficient is 67 $\%$. In the case of the deep strata with a low soil modulus coefficient $\left(H_{1}=8 \mathrm{~m}\right.$ and $K_{0}=$ $15,000)$, the static stiffness coefficient decreases with an increased permanent load. The reduction is small (4\%) and is likely due to the high shear strains beside and under the foundation, which reduces the modulus (see Figure 3 (c)).

- In the pre-resonance frequency range, the system acts as a single degree of freedom system, as described in section 5.1. The real part of the normalized impedance can however be significantly influenced by the permanent load in this range, especially for shallower strata. This is clearly visible in Figure 6 in the case of $H_{1}=2 \mathrm{~m}$ where $\left(k_{j, \text { stat }}-\omega^{2} m_{j, \text { stat }}\right) / k_{z \text {,stat }}$ is less steep for increasing permanent load, which is explained by a larger $k_{z, \text { stat }}$ in relation to $m_{z \text {,stat }}$. In the case of the deep soil, the effect of the permanent load on the pre-resonance impedance is low.

- In the frequency range above the fundamental frequency, the effect of the permanent load can lead to both an increase and a decrease of the normalized impedance at different 

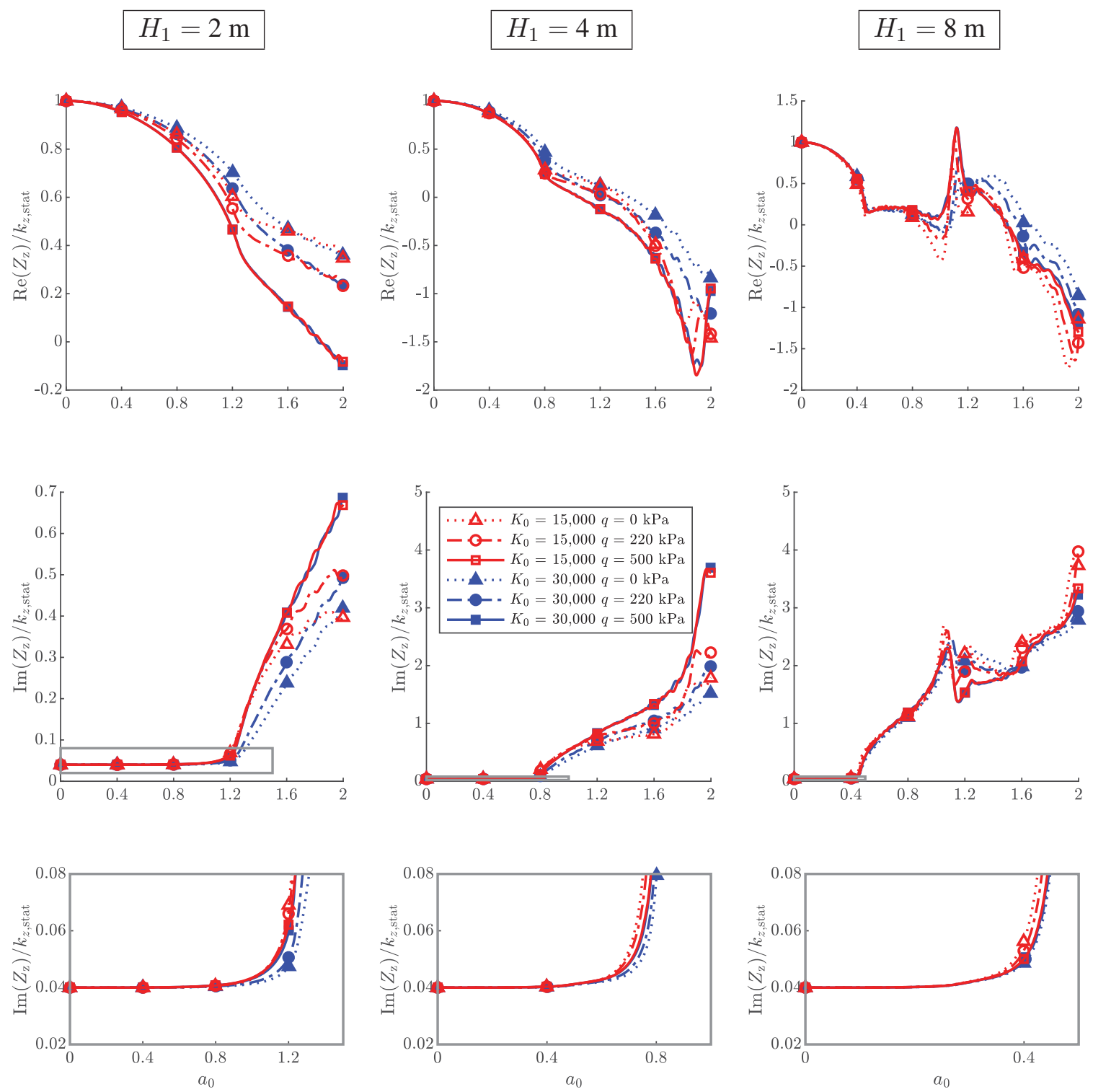

Figure 6: Normalized impedance functions and the effects of the permanent load.
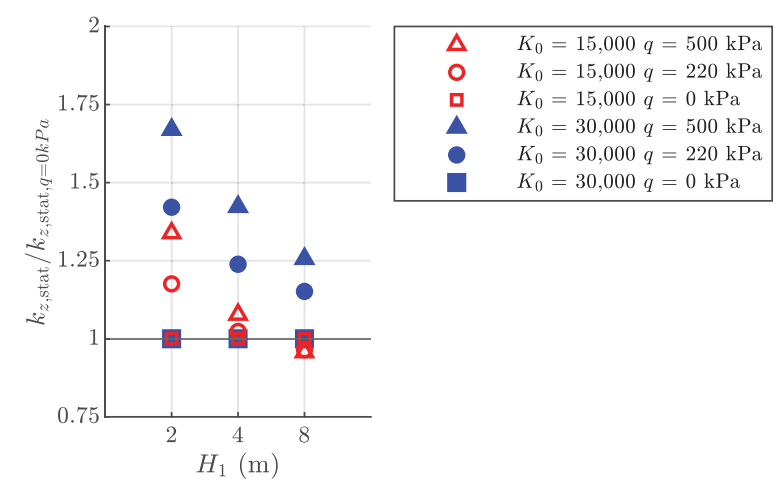

Table 1: Static stiffness coefficients $k_{z, \text { stat }, q=0 k P a}(\mathrm{GN} / \mathrm{m})$.

\begin{tabular}{lrll}
\hline \multirow{2}{*}{$K_{0}$} & \multicolumn{3}{l}{$H_{1}(\mathrm{~m})$} \\
\cline { 2 - 4 } & \multicolumn{1}{c}{2} & 4 & 8 \\
\hline 15,000 & 5.7 & 4.0 & 3.1 \\
30,000 & 11.4 & 7.9 & 6.3 \\
\hline
\end{tabular}

Figure 7: Static stiffness coefficients normalized with $k_{z, \text { stat }, q=0 \mathrm{kPa}}$. 


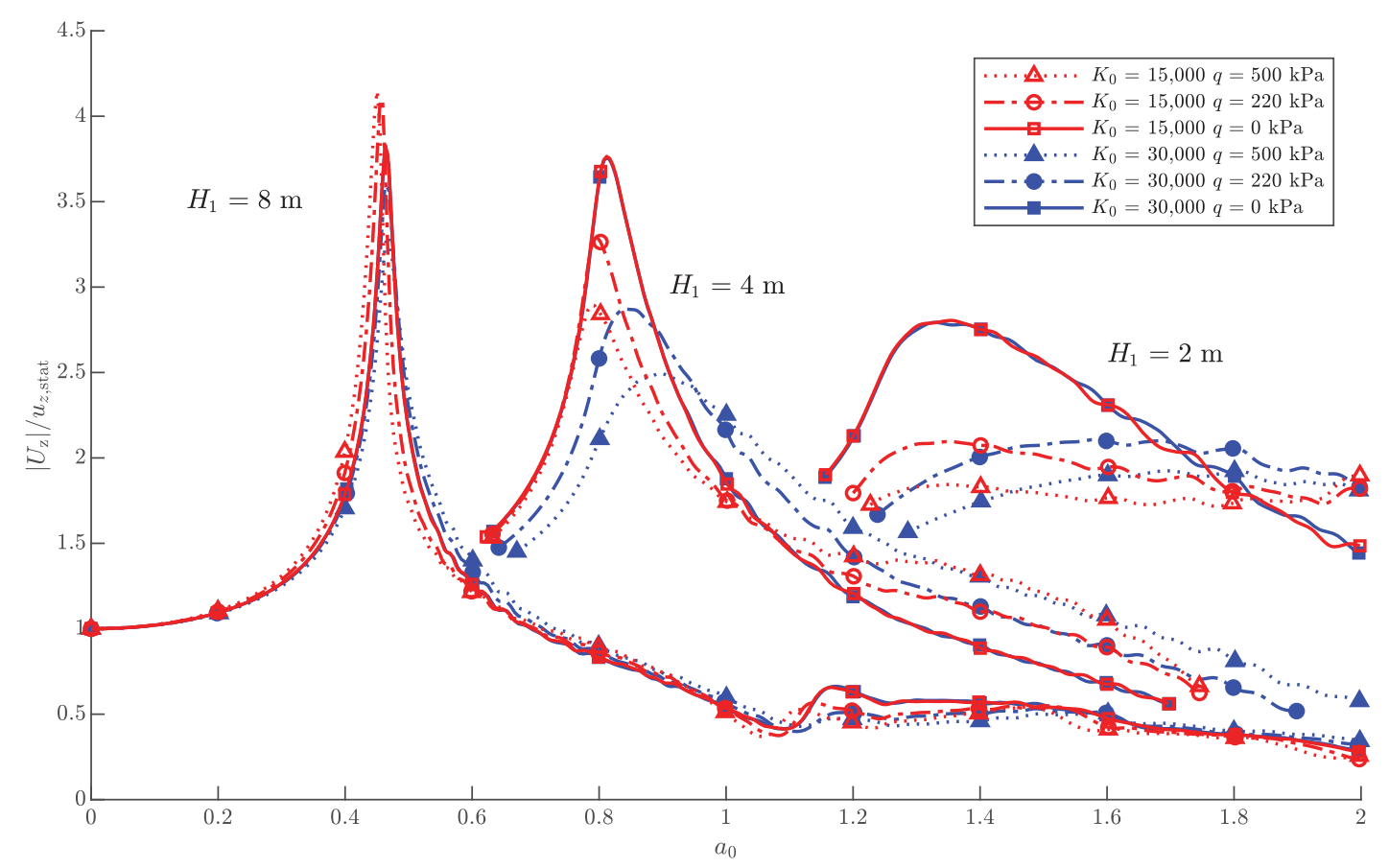

Figure 8: Normalized compliance functions from the parametric study.

frequency ranges. In the case of the shallower strata however, the real part of the normalized impedance is generally increased from the additional permanent load, whereas the imaginary part is decreased. Whereas the effect of the permanent load on the static stiffness coefficients can be quite large, the effect on the normalized impedance functions is relatively small, especially for the deeper strata and at lower frequencies.

- The effect of the permanent load on the fundamental frequency is small, see Fig 8. In the case of $K_{0}=15,000$, the fundamental frequency decreases with an increasing permanent load, whereas it increases in the case of $K_{0}=30,000$. The effect is smaller for deeper soil strata and increases with shallower depths. However, the variations of the fundamental frequency due to the permanent load are small when compared to the ones that are due to the stratum depth and the soil modulus coefficient.

- In most cases, the resonances of the normalized compliance functions decrease with an increasing permanent load, see Figure 8. The decrease is larger in the cases with stiffer and deeper soils. The resonance peaks for these cases are less distinct, whereas the resonance peaks become pointier for deeper and softer soils. The largest reduction of the normalized resonance peak is however observed in the case of the intermediate depth of $H_{1}=4 \mathrm{~m}$ with a stiff soil $K_{0}=30,000$ and is $34 \%$. In the case of the soft and deep soil stratum $\left(H_{1}=8 \mathrm{~m}\right.$ with $\left.K_{0}=15,000\right)$, a small increase of the normalized resonance ( 8 $\%)$ due to the permanent load is observed.

\section{CONCLUSIONS}

The conclusions from the presented studies are summarized in the following main points:

1. The model assumptions can have a great influence on the impedance functions. The con- 
sidered combinations of model assumptions produced normalized impedance functions that are similar in the frequency range below the fundamental frequency. However, in the frequency range above the fundamental frequency, the normalized impedance functions are very different. Further, the static stiffness coefficients of the models differ with more than $100 \%$.

2. The parametric study showed that the permanent load has a significant influence on the normalized impedance functions, especially in the cases of shallow soil strata with high soil modulus coefficients. In most of the cases, an increase of the permanent load leads to an increase of the static stiffness coefficient (with up to $67 \%$ ). However, in the case of the deep and soft soil, it leads to a small decrease of $4 \%$. The normalized impedance functions are in some cases relatively uniform, especially for the deeper strata.

3. The permanent load has a small effect on the fundamental frequency of the soil-foundation system.

\section{ACKNOWLEDGEMENT}

This work was supported financially by the Swedish Transport Administration and the KTH Railway Group.

\section{REFERENCES}

[1] G. Gazetas, Formulas and charts for impedances of surface and embedded foundations, Journal of Geotechnical Engineering, 117(9), 1363-1381, 1991.

[2] J.-G. Sieffert, F. Cevaer, Handbook of impedance functions, Presses Acadeémiques, 1992.

[3] Geological survey of Sweden (SGU), Geology of sweden, Available from: https://www.sgu.se/en/geology-of-sweden/, February 2020.

[4] B. Hardin, V. Drnevich, Shear modulus and damping in soils: Design equations and curves, Journal of the Soil Mechanics and Foundations Division, 98(7), 667-692, 1972.

[5] Prange, B., Resonant column testing of railroad ballast, Technical report, Institute of Soil and Rock Mechanics, University of Karlsruhe, 1981.

[6] Y. Higuchi, Y. Umehara, H. Ohneda, Evaluation of soil properties of the sand deposits under deep sea bed, Proceedings of the 36th annual convention of the Japanese Society for Civil Engineering, 3, 50-51, 1981.

[7] H. B. Seed, I. Idriss, Soil moduli and damping factors for dynamic response analyses (report no. eerc-70/10), Technical report, University of California, 1970.

[8] Swedish Transport Administraion, Trafikverkets tekniska råd för geokonstruktioner-tr geo 13 (swedish), 2016.

[9] J. Jaky, The coefficient of earth pressure at rest, Journal of the Society of Hungarian Architects and Engineers, 7, 355-358, 1944.

[10] R. Dobry, M. Vucetic, Dynamic properties and seismic response of soft clay deposits, Proceedings: International Symposium on Geotechnical Engineering of Soft Soils, 2, 52-57, 1987. 
[11] I. Ishibashi, X. Zhang, Unified dynamic shear moduli and damping ratios of sand and clay, Soil and Foundations, 33(1), 182-191, 1993.

[12] K. M. Rollins, M. D. Evans, N. B. Diehl, W. D. D. III, Shear modulus and damping relationships for gravels, Journal of Geotechnical and Geoenvironmental Engineering, 124(5), 396-405, 1998.

[13] M. B. Darendeli, Development of a New Family of Normalized Modulus Reduction and Material Damping Curves, Dissertation, University of Texas, 2001.

[14] H. B. Seed, R. T. Wong, I. Idriss, K. Tokimatsu, Moduli and damping factors for dynamic analyses of cohesionless soils, Journal of Geotechnical Engineering, 112, 1016-1032, 1986.

[15] J. Lysmer, T. Udaka, C. F. Tsai, H. B. Seed, Flush - a computer program for approximate 3-d analysis of soil-structure interaction problems (report no. pb-259332), Technical report, University of California, 1975.

[16] A. M. Halabian, M. H. El Naggar, Effect of non-linear soil-structure interaction on seismic response of tall slender structures, Soil Dynamics and Earthquake Engineering, 22, 639$58,2002$.

[17] P. A. Costa, R. Calçada, A. S. Cardoso, A. Bodare, Influence of soil non-linearity on the dynamic response of high-speed railway tracks, Soil Dynamics and Earthquake Engineering, 30, 221-235, 2010.

[18] R. Larsson, Information 1 jords egenskaper (swedish), Technical report, SGI - Swedish Geotechnical Institute, 2008.

[19] Swedish Transport Administraion, Trafikverkets tekniska krav för geokonstruktioner-tk geo 13 (swedish), 2014.

[20] G. Gazetas, Vibrational characteristic of soil deposits with variable wave velocity, International Journal for Numerical and Analytical Methods in Geomechanics, 6, 1-20, 1982.

[21] R. Dobry, I. Oweis, A. Urzua, Simplified procedures for estimating the fundamental period of a soil profile, Bulletin of the Seismological Society of America, 66(4), 1293-1321, 1976.

[22] Dassault Systèmes, ABAQUS/Standard: user's manual, Providence, RI, USA., 2017 edition, 2016.

[23] J. Lysmer, R. L. Kuhlemeyer, Finite dynamic model for infinite media, Journal of the Engineering Mechanics Division, pages 859-877, 1969. 